\title{
Fe-Based Nanocrystals in Weak External Magnetic Field
}

\author{
J. SiteK* AND J. DEGMovÁ \\ Department of Nuclear Physics and Technology, Faculty of Electrical Engineering and Information Technology \\ Slovak University of Technology, Ilkovičova 3, 81219 Bratislava, Slovakia \\ Nanocrystalline alloys of $\mathrm{Fe}_{86.5} \mathrm{Zr}_{6.5} \mathrm{Cu}_{1} \mathrm{~B}_{6}$ and $\mathrm{Fe}_{40.5} \mathrm{Co}_{40.5} \mathrm{Nb}_{7} \mathrm{~B}_{12}$ were measured by the Mössbauer \\ spectrometry in the weak external magnetic field of $0.5 \mathrm{~T}$. In these conditions the most sensitive parameters of \\ the Mössbauer spectra are the intensities of 2 nd and 5 th lines and the values of internal magnetic field showed \\ rather small changes. The amorphous part of the sample is more sensitive to the external influence than the \\ nanocrystalline one. The behavior of nanocrystalline alloys under weak external magnetic field was analyzed \\ within influence of interparticles interactions on the magnetic parameters.
}

PACS numbers: $33.45 .+\mathrm{x}, 61.46 .-\mathrm{w}, 75.50 . \mathrm{Kj}$

\section{Introduction}

For investigation of structural and magnetic properties of nanomaterials in order to optimize their technology a number of techniques can be used. Among them the Mössbauer spectroscopy is one of more suitable tool [1]. Up to now magnetic parameters derived from the Mössbauer spectra of nanocrystalline materials were usually measured as a function of temperature. In most of the cases the spectra were measured in a strong external magnetic field of about several T [2,3]. An influence of weak magnetic field with the strength of less than $1 \mathrm{~T}$ on the shape of the Mössbauer spectra of nanocrystalline materials were measured only occasionally $[4,5]$.

In the present work we focused at the influence of weak magnetic field on the Fe-based nanocrystalline of Nanoperm and Hitperm type.

\section{Experimental}

The nominal composition of the ribbon-shaped amorphous specimens was $\mathrm{Fe}_{86.5} \mathrm{Zr}_{6.5} \mathrm{Cu}_{1} \mathrm{~B}_{6}$ and $\mathrm{Fe}_{40.5} \mathrm{Co}_{40.5} \mathrm{Nb}_{7} \mathrm{~B}_{12}$. Annealing was carried out in vacuum at the temperature range from 550 to $600^{\circ} \mathrm{C}$ for $1 \mathrm{~h}$. The Mössbauer spectra were collected in transmission geometry by a conventional constant acceleration spectrometer with a ${ }^{57} \mathrm{Co}(\mathrm{Rh})$ source. The Mössbauer measurements were performed in weak external magnetic field up to $0.5 \mathrm{~T}$, which was oriented perpendicular to the sample plane. All spectra were measured at room temperature. The spectra were evaluated by the Normos program [6], which allows simultaneous treatment of crystalline and residual amorphous phase by means of individual lines and distribution of hyperfine components.

* corresponding author; e-mail: jozef.sitek@stuba.sk

\section{Results and discussion}

The Mössbauer spectra of nanocrystalline samples measured with and without application of weak magnetic field are shown in Fig. 1 and Fig. 2. The applied fitting model consists of two main components, i.e. of one consisting of lines attributed to the Fe atoms situated in the bulk of nanocrystalline grains and one individual subspectrum resulting from the distribution of hyperfine parameters ascribed to amorphous residual matrix. Table summarizes the behavior of most sensitive parameters under influence of weak magnetic field. The results showed that the values of second and fifth line intensities ratio $\left(A_{23}\right)$ exhibit rather significant changes while the values of average internal magnetic field $(B)$ changed only slightly.

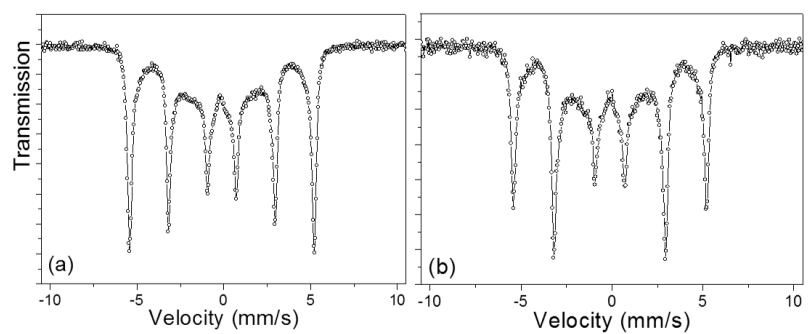

Fig. 1. The Mössbauer spectra of nanocrystalline Nanoperm (a) without and (b) with application of magnetic field.

In the case of nanocrystalline samples the three magnetic fields should be taken into account, namely internal magnetic field of crystalline component, average magnetic field of amorphous component and weak external magnetic field. It must be also taken into account that all these three fields interact together and have common influence on the orientation of the magnetic domains. We also cannot exclude the presence of interactions among the particles because these interparticles interactions in- 


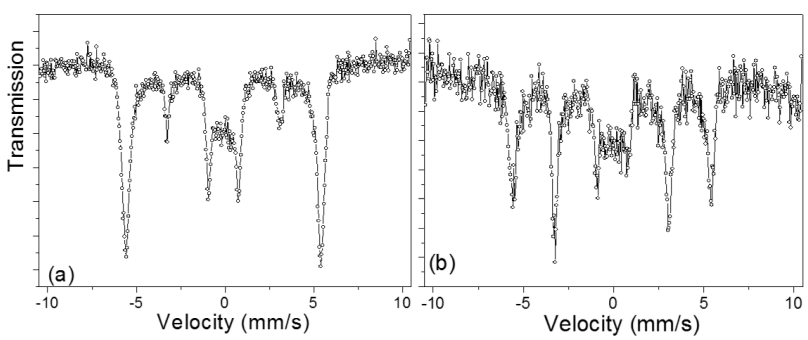

Fig. 2. The Mössbauer spectra of nanocrystalline Hitperm (a) without and (b) with application of magnetic field.

TABLE

The Mössbauer parameters of nanocrystalline Nanoperm $\left(\mathrm{Fe}_{86.5} \mathrm{Zr}_{6.5} \mathrm{Cu}_{1} \mathrm{~B}_{6}\right)$ and Hitperm $\left(\mathrm{Fe}_{40.5} \mathrm{Co}_{40.5} \mathrm{Nb}_{7} \mathrm{~B}_{12}\right)$ alloys, where $A_{23 \mathrm{am}}, B_{\mathrm{am}}$ and $\%$ am are parameters of amorphous subspectra and $A_{2 x}, B_{\mathrm{cr}}$ and $\%_{\mathrm{cr}}$ are parameters of crystalline subspectra. (m) indicates measurement in magnetic field.

\begin{tabular}{c|c|c|c|c|c|c}
\hline \hline Sample & $A_{23 \mathrm{~m}}$ & $B_{\mathrm{am}}[\mathrm{T}]$ & $\%_{\mathrm{am}}$ & $A_{2 x}$ & $B_{\mathrm{cr}}[\mathrm{T}]$ & $\%_{\mathrm{cr}}$ \\
\hline Nanoperm & 2.4 & 19.7 & 62 & 2 & 33 & 38 \\
Nanoperm(m) & 3.7 & 18.7 & 60 & 3.3 & 33 & 40 \\
Hitperm & 0.4 & 21.6 & 63 & 2.7 & 34 & 37 \\
Hitperm(m) & 2.6 & 23.8 & 72 & 3.8 & 34 & 28
\end{tabular}

fluence quite strongly at relaxation and magnetization processes.

Our results of nanocrystalline Nanoperm alloy exhibits sensitivity to weak external magnetic field at both components. The intensities ratio $A_{23}$ of crystalline part increases by about 1.3 which gives us the evidence that orientation of magnetic domains turns more into direction of the ribbon plane. Magnetic moments have similar behavior in amorphous part where $A_{23}$ changes also about value 1.3. The corresponding value of average internal magnetic field exhibits small changes in amorphous component and no changes in crystalline component. Orientation of the local moments is included in the parameter $A_{23}$.

Hitperm exhibits also similar behavior as Nanoperm alloy. However, the changes in parameters are more pronounced. The $A_{23}$ parameter of amorphous part increased about 2.2 while that for crystalline part about 1.1. This indicates that the magnetic domains turn into the same direction as in the case of Nanoperm. Average internal magnetic field for amorphous component changed by about $2 \mathrm{~T}$ and no changes were observed for crystalline part. These results indicate that the amorphous part of the sample is more sensitive to the external influence than nanocrystalline one.
We suppose in both cases that the original magnetic interactions (without influence of external field) in amorphous part are weaker than in crystalline one, which could explain such a behaviour.

According to [5] the presence of interparticles interaction has different influence at relaxation phenomena. However, in our case, including mean field interparticle interaction the magnetization will be dependent on magnetic anisotropy and on the angle between direction of magnetization and external magnetic field. This could be reflected also on the shape of the Mössbauer spectrum.

Our previous results confirmed that in the case of Finemet alloys having small anisotropy, the influence of weak external magnetic field was negligible [7]. It should be noted that magnetic properties of Nanoperm as anisotropy are very sensitive to impurity and technology preparation. We suppose that this is a reason of higher sensitivity at weak external magnetic field. Similar behaviour is exhibited also in Hitperm alloy.

\section{Conclusion}

Our results indicate that weak external magnetic field has influence on the direction of magnetization, and this is reflected in parameters of the Mössbauer spectra. For such a study nanocrystalline alloys are suitable because they are sensitive to anisotropy changes.

\section{Acknowledgments}

This work was partially supported by VEGA 1/0606/09.

\section{References}

[1] B.F.O. Costa, G. Le Caër, B. Luyssaerta, J. Alloys Comp. 350, 36 (2003).

[2] X.Y. Sun, L. Zhen, C.Y. Xu, L.X. Lv, W.Z. Shao, X.D. Sun, Mater. Lett. 63, 64 (2009).

[3] M. Reissner, E. Bauer, W. Steiner, P. Rogl, J. Magn. Magn. Mater. 272-276, 813 (2004).

[4] M.A. Polikarpov, V.M. Cherepanov, M.A. Chuev, S.Yu. Shishkov, S.S. Yakimov, in: ICAME Proc., Institute of Solid State Physics, TU Vienna, Vienna 2009, p. 297.

[5] M.A. Chuev, J. Phys., Condens. Matter 20, 505201 (2008).

[6] R.A. Brand, NORMOS program (1997), unpublished.

[7] J. Degmova, J. Sitek, AIP Conf. Proc., accepted for publication. 